\title{
Cornification of nail keratinocytes requires autophagy for bulk degradation of intracellular proteins while sparing components of the cytoskeleton
}

\author{
Karin Jaeger ${ }^{1}$. Supawadee Sukseree ${ }^{1}$ - Shaomin Zhong ${ }^{1}$ - Brett S. Phinney ${ }^{2}$. Veronika Mlitz ${ }^{1}$ Maria Buchberger ${ }^{1}$. \\ Marie Sophie Narzt ${ }^{1,3} \cdot$ Florian Gruber $^{1,3} \cdot$ Erwin Tschachler $^{1} \cdot$ Robert H. Rice $^{4} \cdot$ Leopold Eckhart $^{1}$ (])
}

Published online: 14 December 2018

(c) The Author(s) 2018

\begin{abstract}
Epidermal keratinocytes undergo cornification to form the cellular building blocks of hard skin appendages such as nails and the protective layer on the surface of the skin. Cornification requires the cross-linking of structural proteins and the removal of other cellular components to form mechanically rigid and inert corneocytes. Autophagy has been proposed to contribute to this intracellular remodelling process, but its molecular targets in keratinocytes, if any, have remained elusive. Here, we deleted the essential autophagy factor Atg7 in K14-positive epithelia of mice and determined by proteomics the impact of this deletion on the abundance of individual proteins in cornified nails. The genetic suppression of autophagy in keratinocytes resulted in a significant increase in the number of proteins that survived cornification and in alterations of their abundance in the nail proteome. A broad range of enzymes and other non-structural proteins were elevated whereas the amounts of cytoskeletal proteins of the keratin and keratin-associated protein families, cytolinker proteins and desmosomal proteins were either unaltered or decreased in nails of mice lacking epithelial autophagy. Among the various types of non-cytoskeletal proteins, the subunits of the proteasome and of the TRiC/CCT chaperonin were most strongly elevated in mutant nails, indicating a particularly important role of autophagy in removing these large protein complexes during normal cornification. Taken together, the results of this study suggest that autophagy is active during nail keratinocyte cornification and its substrate specificity depends on the accessibility of proteins outside of the cytoskeleton and their presence in large complexes.
\end{abstract}

Keywords Cornification $\cdot$ Autophagy $\cdot$ Proteomics $\cdot$ Keratinocytes $\cdot$ Keratin $\cdot$ Nail

Electronic supplementary material The online version of this article (https://doi.org/10.1007/s10495-018-1505-4) contains supplementary material, which is available to authorized users.

Robert H. Rice

rhrice@ucdavis.edu

$\triangle$ Leopold Eckhart

leopold.eckhart@meduniwien.ac.at

1 Research Division of Biology and Pathobiology of the Skin, Department of Dermatology, Medical University of Vienna, Lazarettgasse 14, 1090 Vienna, Austria

2 Proteomics Core Facility, UC Davis Genome Center, University of California, Davis, CA, USA

3 Christian Doppler Laboratory on Biotechnology of Skin Aging, Vienna, Austria

4 Department of Environmental Toxicology, University of California, One Shields Avenue, Davis, CA 95616-8588, USA

\section{Introduction}

Cornification is a special form of programmed cell death that does not lead to the disintegration or phagocytosis of dead cells but to the conversion of keratinocytes into "corneocytes" that are integrated into hard skin appendages such as nails and into the protective cornified layer (stratum corneum) on the surface of the epidermis (Fig. 1a, b) [1-4]. Corneocytes are densely packed with keratin intermediate filaments and associated proteins that are cross-linked by transglutamination and disulfide bonds. Other intracellular components of keratinocytes, including the nucleus, are removed whereas cell junctions via desmosomes are maintained during cornification (Fig. 1c). The proteolytic cleavage of cell junctions in the superficial layers of the stratum corneum causes the shedding of epidermal corneocytes 


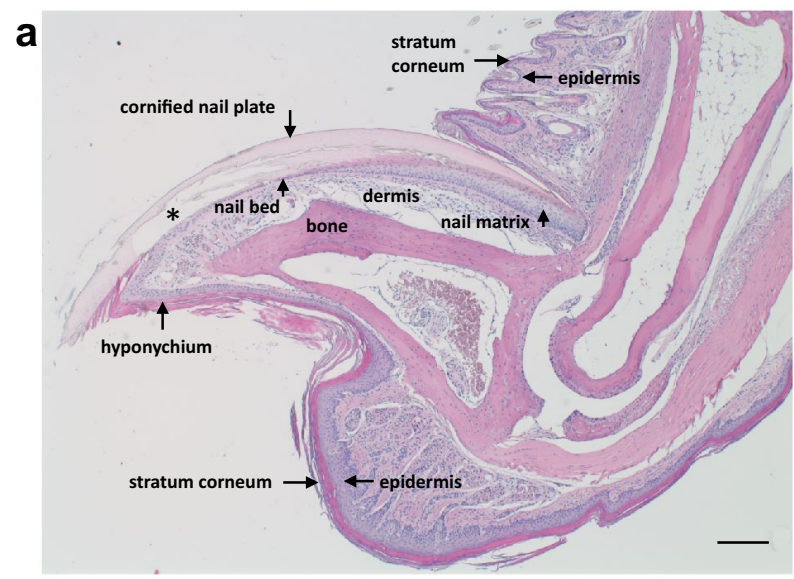

b

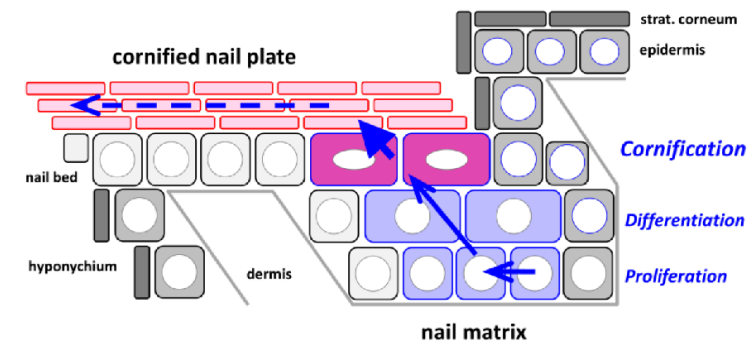

C

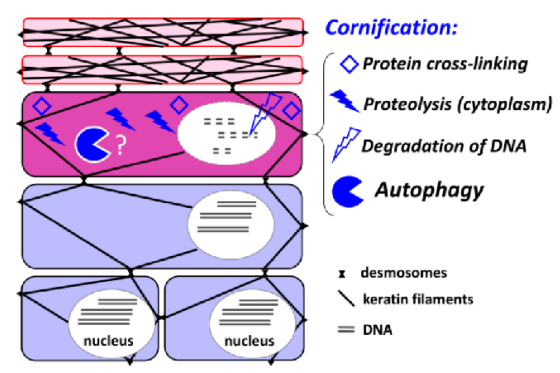

Fig. 1 Cornification of nail keratinocytes involves intracellular remodelling. a Hematoxylin and eosin (H\&E) staining of a sagittal section through a mouse toe. Parts of the nail plate $(*)$ have been lost in the course of sample processing. Size bar, $200 \mu \mathrm{m}$. b Schematic depiction of keratinocyte differentiation in the nail matrix (indicated by arrows). c Schematic depiction of intracellular remodelling during keratinocyte differentiation and cornification. The aim of the study is to determine whether autophagy in cornifying keratinocytes influences the proteome of the cornified nail

(desquamation), and continued presence of intercellular connections facilitates the growth of cornified nails.

The degradative processes during cornification have similar effects as autophagy, i.e. the ubiquitous intracellular program for delivery of cellular components to lysosomes for digestion [5-8]. Autophagy was suggested to remove organelles during terminal differentiation of keratinocytes [4, 9-12] and specifically to degrade the nucleus (nucleophagy) [13]. However, the deletion of autophagy-related 7 (Atg7), which is essential for macroautophagy, the main form of autophagy in mammalian cells, in keratin K14-positive epithelial cells (Atg $7^{f l f} \mathrm{~K} 14-\mathrm{Cr}$ ) did not impair DNA degradation in the interfollicular epidermis of mice [14]. Suppression of epithelial autophagy even led to premature degradation of the nucleus in sebocytes $[15,16]$. In addition, the stratum corneum of $\mathrm{Atg} 7^{f / f} \mathrm{~K} 14-\mathrm{Cre}$ mice was thickened [14], and the protein turnover in thymic epithelial cells [17], sweat glands [18], Merkel cells [19], and sebaceous glands [20] was altered. The autophagy adapter and substrate proteins microtubule-associated proteins $1 \mathrm{~A} / 1 \mathrm{~B}$ light chain $3 \mathrm{~A}$ (LC3) and sequestosome 1 (Sqstm1)/p62 were elevated in isolated epidermal keratinocytes upon deletion of autophagy genes [15, 21], but a comprehensive catalog of keratinocyte proteins targeted by autophagy has remained elusive.

In the present study, we determined the protein composition of the cornified nails of $\mathrm{Atg} 7^{\mathrm{flf}} \mathrm{K} 14$-Cre mice and autophagy-competent control mice. We provide in vivo evidence for the hypothesis that autophagy shapes the proteome of cornifying nail keratinocytes.

\section{Materials and methods}

\section{Mice}

The generation of $\mathrm{Atg} 7^{\mathrm{flf}} \mathrm{K} 14-\mathrm{Cre}(\mathrm{KO})$ mice by crossing Atg $7^{f f f}$ (WT) mice [22] and K14-cre mice, strain Tg(KRT14cre) 1 Amc/J (Jackson Laboratories, Bar Harbour, ME) on a B6/CBA background has been reported previously [14, 17]. GFP-LC3 transgenic mice [23] were crossed with Atg $7^{f l f}$ K14-Cre mice to obtain Atg $7^{f l f} G F P-L C 3$ and Atg $7^{f l f}$ K14-Cre GFP-LC3 mice. Genotyping and maintenance of mice was done as reported [14]. Animal procedures were performed according to the guidelines of the Ethics Review Committee for Animal Experimentation of the Medical University of Vienna, Austria (approval number BMWF-66.009/0124-II/10b/2010).

\section{Nail morphology, histology, immunofluorescence and fluorescence analysis}

Toes were cut off the feet of sacrificed WT and KO mice and photographed under a Leica MZ 16 stereomicroscope using a Leica IC 3D CCD camera (Leica Microsystems, Wetzlar, Germany). Tissues were fixed in formaldehyde, embedded in paraffin, sectioned and stained with hematoxylin and eosin (H\&E). DNase1L2 was detected by immunofluorescence labeling according to a published protocol [24]. DNA fragments were detected in formaldehyde-fixed tissue sections by terminal deoxynucleotidyl transferase dUTP nick end labeling (TUNEL) according to a published protocol [25]. Nuclei were counter-labeled with Hoechst dye. The presence of recombinant GFP-LC3 
puncta was investigated on cryosections using an LSM700 confocal laser microscope (Zeiss) [17].

\section{Sample preparation for proteomic analysis}

Immediately after mice were sacrificed, the nails were cut off from toes. Only non-pigmented nails were used. The adhering tissue was removed by heating the samples in $2 \%$ SDS in phosphate-buffered (PBS) for $1 \mathrm{~h}$. Subsequently the nails were washed in PBS 3 times followed by final washes in $70 \%$ ethanol and $100 \%$ ethanol. The nails were air-dried and stored at room temperature prior to further processing. Samples from each mouse were processed separately. The nails were incubated for $1.5 \mathrm{~h}$ at $70{ }^{\circ} \mathrm{C}$ in $2 \% \mathrm{SDS}, 0.1 \mathrm{M}$ sodium phosphate ( $\mathrm{pH} 7.8$ ), $20 \mathrm{mM}$ dithioerythritol, and then alkylated with $40 \mathrm{mM}$ iodoacetamide. Total protein was recovered by ethanol precipitation, rinsed in $67 \%$ ethanol and digested with reductively methylated trypsin [26] in fresh $0.05 \mathrm{M}$ ammonium bicarbonate containing 5\% acetonitrile for 3 days at room temperature.

\section{LC-MS/MS analysis}

Peptides were separated on a Michrom Paradigm HPLC (Michrom Corporation) with a Michrom Easy-Spray source. The digested peptides were reconstituted in $2 \%$ acetonitrile $/ 0.1 \%$ trifluoroacetic acid and roughly $3 \mu \mathrm{g}$ of each sample was loaded onto a 100 micron $\times 25 \mathrm{~mm}$ Magic C18 $100 \AA 5 \mathrm{U}$ reversed-phase trap where they were desalted online before being separated on a 200 micron $\times 150 \mathrm{~mm}$ Magic C18 $200 \AA 3 \mathrm{U}$ reversed-phase column. Peptides were eluted using a gradient of $0.1 \%$ formic acid (A) and $100 \%$ acetonitrile (B) with a flow rate of $300 \mathrm{~nL} / \mathrm{min}$. A $60 \mathrm{~min}$ gradient was run with 5-35\% B over $110 \mathrm{~min}, 35-80 \%$ B over $3 \mathrm{~min}, 80 \%$ B for $1 \mathrm{~min}$, $80-5 \%$ B over $1 \mathrm{~min}$, and finally held at $5 \%$ B for $5 \mathrm{~min}$.

Mass spectra were collected on an Orbitrap Q Exactive mass spectrometer (Thermo Fisher Scientific) in a datadependent mode with one MS precursor scan followed by $15 \mathrm{MS} / \mathrm{MS}$ scans. A dynamic exclusion of $15 \mathrm{~s}$ was used. MS spectra were acquired with a resolution of 70,000 and a target of $1 \times 10^{6}$ ions or a maximum injection time of $30 \mathrm{~ms}$. MS/MS spectra were acquired with a resolution of 17,500 and a target of $5 \times 10^{4}$ ions or a maximum injection time of $50 \mathrm{~ms}$. Peptide fragmentation was performed using higher-energy collision dissociation (HCD) with a normalized collision energy (NCE) value of 27. Unassigned charge states as well as +1 and ions $>+5$ were excluded from MS/MS fragmentation.

\section{Peptide spectrum matching}

MS/MS data were analyzed using using X! Tandem (http:// www.thegpm.org; version CYCLONE (2013.02.01.1)) assuming the digestion enzyme trypsin. $\mathrm{X}$ ! Tandem was set up to search the Uniprot mouse database (8 July 2013, 86,032 entries) appended to an identical but reversed peptide database for estimating false discovery rate. $\mathrm{X}$ ! Tandem was searched with a fragment ion tolerance of $20 \mathrm{ppm}$ (monoisotopic) and a parent ion tolerance of $20 \mathrm{ppm}$ (monoisotopic). Iodoacetamide derivative of cysteine was specified in X! Tandem as a fixed modification. Variable Modifications: N-terminal Glu $\rightarrow$ pyro-Glu and loss of ammonia, deamidation of $\mathrm{N}$ and $\mathrm{Q}$, oxidation and dioxidation of $\mathrm{M}$ and $\mathrm{W}$, and acetylation of the N-terminus. Scaffold (version Scaffold_4.8.4, Proteome Software, Inc., Portland, OR) was used to validate MS/MS based peptide and protein identifications. Protein probabilities were assigned by the Protein Prophet algorithm [27]. Peptide FDR: 0.5\% (Decoy), Protein FDR: $2.8 \%$ (Decoy). Proteins that contained peptides that could not be distinguished by MS/MS analysis were grouped for parsimony. Peptides potentially derived from contamination by human material were removed from the analysis.

\section{Intensity-based absolute quantification (iBAQ)}

iBAQ values of individual proteins were used to calculate the relative molar amount of each protein [28]. iBAQ values were calculated by searching the mass spectrometry data with Maxquant (1.5.7.4) against the Uniprot Mouse proteome (3AUP000000589, Dec 2017) using Maxquant's default settings with the exception that match between runs was turned on. Peptides and proteins were filtered with a decoy false discovery rate of $1 \%$. Maxquant MS1 intensity values and identification results were imported into Scaffold (version 4.82) and Scaffold was used to calculate the iBAQ values for each sample. To this end, average calculated values for each protein were normalized to the total values for a given genotype.

\section{Data availability}

Mass spectrometry and proteomics data were uploaded to the Massive Proteomics data repository (massive.ucsd.edu) and Proteome Exchange (http://www.proteomexchange .org/) and are avalaible under the identification numbers MSV000082333 and PXD009663, respectively.

\section{Reverse transcription polymerase chain reactions (RT-PCR)}

The two biggest claws of each paw were pulled out immediately after sacrificing mice ( 8 claws per animal). The 
claws and the adhering nail matrix were incubated in $1 \mathrm{ml}$ TriFast (VWR, Radnor, PA) for $1 \mathrm{~h}$ at $4{ }^{\circ} \mathrm{C}$. RNA was extracted from the nail matrix and, as control, of homogenized sole skin, using TriFast according to the manufacturer's instructions and reverse-transcribed to cDNA with the Iscript ${ }^{\mathrm{TM}}$ kit (Biorad, Hercules, CA).

Transcripts of Atg7 and the housekeeping gene Beta2 microglobulin $(B 2 m)$ were amplified using DreamTaq ${ }^{\mathrm{TM}}$ DNA polymerase (Thermo Scientific, Waltham, CA) and the primer pairs Atg7_f, agcttggctgctacttctgc and Atg7_r, tcattcatgcggtcatcact, and B2m_f, attcacccceactgagactg and B2m_r, tgctatttctttctgcgtgc. B2m was previously established as a house-keeping gene equally expressed in WT and KO keratinocytes [29]. The PCR products were electrophoresed through $1.5 \%$ agarose gels and labeled with GelRed nucleic acid stain (Biotium, Fremont, CA).

Transcripts of the following genes were quantified by qPCR, using the LightCycler $\rightarrow$ (LC480) technology (Roche Applied Science, Basel, Switzerland) and the LightCycler 480 DNA SYBR Green I Master Kit (Roche Applied Science) according to a published protocol [30] with the indicated primer pairs: Aloxe3 (Aloxe3_f, aggcacctgcctacaaacag and Aloxe3_r, atcagtgggcagaaagatgg), Cct3 (Cct3_f, agtcatcagtcggtggtcct and Cct3_r, taatatagcggcgcatcctt), Hspa5 (Hspa5_f, agcgacaagcaaccaaagat and Hspa5_r, atgaccegctgatcaaagtc), Krt86 (Krt86_f, ggagcagaggttgtgtgagg and Krt86_r, tgcagcattgtgacctccta), Lrrc15 (Lrrc15_f, ccgcctccttcttattgacc and Lrrc15_r, ggagttcggtgatgtgtgtg), Lygl (Lyg1_f, tggggatgctatggaaacat and Lyg1_r, cgaccacatagttgcctcct), Pdia3 (Pdia3_f, tatgatgggectaggactgc and Pdia3_r, tgctggctgcttttaggaat), Pdia6 (Pdia6_f, gccaccatgaatcaggttct and Pdia6_r, ctctggaggaggggcattat), and Psmd2 (Psmd2_f, agcaggagctgtctgaggag and Psmd2_r, ggacgcagaaatttgagagg). The relative expression of each gene was normalized to that of $B 2 \mathrm{~m}$, which was amplified with the primer pair indicated above and quantified using a mathematical model described previously [31].

\section{Statistical analysis}

Data are presented as mean \pm standard deviation (SD). Standard deviations of subtractions (KO-WT) and divisions (KO/WT) of means were calculated according to the rules of error propagation. The significance of difference between groups was determined with the two-tailed t-test. To reduce effects of multiple testing, we focused our comparative analysis on a small number of protein groups instead of a large number of individual proteins and based major conclusions only on differences supported by $p$ values smaller than 0.01 instead of the standard $p<0.05$.

\section{Results}

\section{Atg7-deficiency does not abrogate differentiation of nail keratinocytes in $\mathrm{Atg}^{\mathrm{flf}} \mathrm{K14}$-Cre mice}

As the constant turnover of proteins in living cells limits the power of proteomic analysis, we chose the cornified nails as a model system in which the proteome of nail-forming cells is "frozen" by protein cross-linking. To study the role of autophagy in the cornification process, we compared Atg $7^{f f f}$ (wildtype, WT) and $\mathrm{Atg} 7^{\mathrm{flf}} \mathrm{K} 14$-Cre (knockout, KO) mice in which the essential autophagy factor $\operatorname{Atg} 7$ is deleted in keratinocytes [14].

To determine the expression of Atg7 in nail keratinocytes, we extracted RNA from cells of the nail matrix of Atg $7^{\text {flf }}$ (wildtype, WT) and $\mathrm{Atg} 7^{\text {flf }} \mathrm{K14}$-Cre (knockout, KO) mice and performed RT-PCR. Atg7 was readily detected in the nail matrix of WT but not of KO mice, confirming efficient inactivation of $\operatorname{Atg} 7$ expression in the cells that form nails (Fig. 2a, b). To monitor autophagosomes in situ, the transgenic reporter protein GFP-LC3 was introduced into a subline of $A t g 7^{f l f}$ and $A t g 7^{f l f}$ K14-Cre mice. GFP-LC3labelled autophagosomes were detected in the nail matrix of WT (Atg $\left.7^{f f f} G F P-L C 3\right)$ mice (Suppl. Fig. S2a, b) but not a
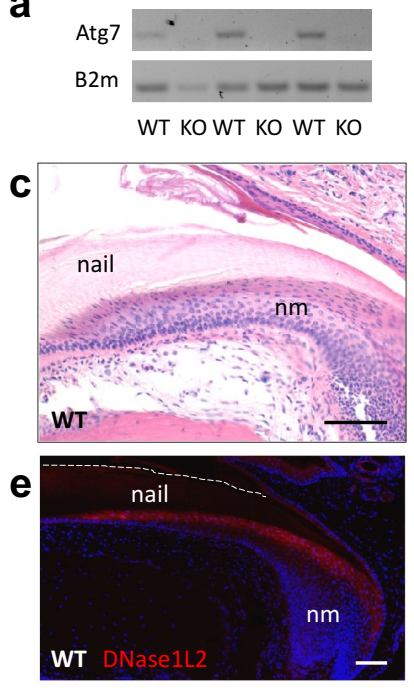
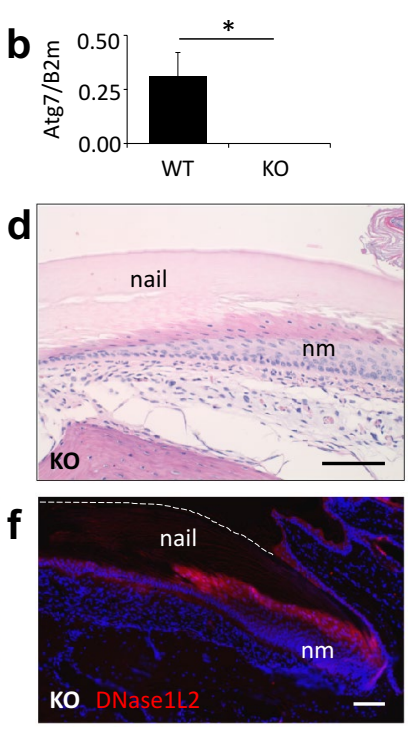

Fig. 2 K14-Cre-mediated deletion of Atg7 in the nail matrix is compatible with histologically normal nail formation. a RT-PCR analysis of $A t g 7$ and $B 2 m$ expression in the nail matrix of mice carrying floxed Atg7 alleles either in the absence (WT) or presence (KO) of K14Cre. b RT-PCR band intensities were quantified. The graph shows the means of the intensity ratios $A \operatorname{tg} 7 / B 2 m$, error bars show standard deviations. The ratio was significantly reduced $(p<0.05,2$-sided $\mathrm{t}$ test, *) in Atg $7^{f l f}$ K14-Cre (KO) mice. c, d H\&E staining of the nail matrix (nm) of $A t g 7^{f l f}$ (WT) and Atg $7^{f l f}$ K14-Cre (KO) mice. e, f Immunofluorescence labeling of DNase1L2 (red) in the nail matrix. Scale bars, $100 \mu \mathrm{m}$ 
in the nail matrix of $\mathrm{KO}\left(\operatorname{Atg} 7^{\mathrm{flf}} \mathrm{K} 14-\mathrm{Cre}\right.$ GFP-LC3) mice (Suppl. Fig. S2c), confirming the suppression of autophagy upon epithelial deletion of Atg7. Terminal deoxynucleotidyl transferase dUTP nick end labeling (TUNEL) showed that the blockade of autophagy did not lead to premature cell death in differentiating nail keratinocytes (Suppl. Fig. S3). TUNEL-positive DNA 3'-OH ends remained confined to the layer of cornifying keratinocytes adjacent to the nail plate in both WT and KO mice (Suppl. Fig. S3).

WT and KO mice were maintained under standard housing conditions in which the nails are exposed to only weak mechanical stress. The nails of both mouse genotypes had the same shape, suggesting that growth and wear and tear were not altered by the deletion of Atg7 (Suppl. Fig. S1). $\mathrm{H} \& \mathrm{E}$ staining of sagittal sections through the nail unit showed the normal stages of keratinocyte differentiation including a cornification-associated change from eosinophilic keratinocytes to essentially unstained nail corneocytes in both WT and KO mice (Fig. 2c, d). Immunofluorescence labeling demonstrated normal expression of the terminal differentiation marker DNase1L2 in the nail matrix and spatial correlation of DNase1L2 expression with the breakdown of nuclear DNA in nails of both genotypes (Fig. 2e, f; Suppl. Fig. S3) [32, 33]. Gene expression analysis by quantitative RT-PCR showed that expression of DNase1L2 and other genes in the nail matrix was not significantly different between WT and KO mice (Suppl. Fig. S4). Thus deletion of $\operatorname{Atg} 7$ was compatible with the normal execution of the genetic program of nail keratinocyte differentiation.

\section{Suppression of autophagy leads to an increase in protein diversity and a decrease of the fraction of cytoskeletal proteins in nails}

To determine the role of autophagy in the re-shaping of the proteome during cornification, $3 \mathrm{WT}$ and $3 \mathrm{KO}$ mice were sacrificed and cornified nails were prepared, taking care to remove all surrounding tissue. The protein composition of the nails of each mouse was determined using a proteomics approach described previously for cornified skin and appendages [34, 35]. In brief, the proteins were vigorously extracted with SDS buffer containing dithioerythritol to break disulfide bonds followed by digestion with trypsin, separation by reversed-phase chromatography and tandem mass spectrometry (MS). Relative amounts of a given protein in different samples were compared by intensity-based absolute quantification (iBAQ) [28].

In nails of WT mice, a total of $518 \pm 11($ mean \pm SD) proteins were identified whereas $633 \pm 2$ proteins were identified in nails of KO mice (Suppl. Table S1), representing an increase of the protein diversity by more than
$20 \%$ ( $p<0.01$, t test). Similarly, the number of highly abundant proteins (iBAQ $>10^{7}$ ) was increased by $20 \%$ in KO nails relative to WT nails (WT, $\mathrm{n}=284 ; \mathrm{KO}, \mathrm{n}=345$; $p<0.01$ ).

To determine the influence of autophagy on the abundance of different proteins in cornified nails, we compared the iBAQ values of protein groups and individual proteins in the nails from WT and KO mice. First, we categorized proteins according to their contribution to the primary feature of nails, that is mechanical resilience. This characteristic trait of nails depends on stable protein structures that consist of the keratin cytoskeleton and stable cell junctions. As a proxy, we calculated the sum of iBAQ values of "structural proteins" including keratins, keratin-associated proteins (KRTAPs), and cell junction proteins (desmosome and cytolinker proteins), relative to all other proteins, here termed "non-structural proteins" (Table 1). These structural proteins accounted for $79 \pm 4 \%$ (mean \pm SD) of the iBAQ values of WT nails but only for $71 \pm 2 \%$ of the iBAQ values of nails formed by Atg7 KO keratinocytes (Fig. 3a). Conversely, $21 \pm 4 \%$ and $29 \pm 2 \%$ iBAQ values corresponded to non-structural proteins in WT and KO nails, respectively, with the difference being statistically significant. The ratio of $\mathrm{BAQ}$ values of non-structural versus structural proteins was increased from $0.26 \pm 0.06$ in WT nails to $0.42 \pm 0.03$ in $\mathrm{KO}$ mice $(p<0.01)$.

At the level of individual proteins, the autophagy adapter and substrate protein LC3 (microtubule-associated proteins 1A/1B light chain 3A, MAP1LC3A) was significantly increased in KO nails (Fig. 3b), confirming an effective blockade of autophagic turnover in Atg7-deficient keratinocytes. The abundance of all structural proteins, as illustrated by the example of KRTAP6-5 in Fig. 3c, was uniformly decreased in KO mouse nails (Suppl. Table S1) whereas Atg7-dependent changes in abundance of nonstructural proteins were more variable, as will be described in detail in the next section, and included multiple cases of more than 10-fold elevations (Suppl. Table S1). DNase1L2, which is expressed in differentiated keratinocytes of both genotypes (Fig. 2e, f), was absent in the proteome of cornified WT nails, indicating degradation of DNase1L2 during cornification, but abundantly present in $\mathrm{KO}$ nails (Fig. 3d). The increase of DNase1L2 protein levels in KO nails was not caused by an elevation of Dnase 112 gene expression in the nail matrix, because equal amounts of DNase 1L2 mRNA were detected by quantitative RT-PCR in WT and KO mice (Fig. 3e). Likewise, other changes in protein abundance were not accompanied by changes in the levels of corresponding mRNAs (Suppl. Fig. S4), suggesting that the K14-Cre-mediated deletion of $\operatorname{Atg} 7$ altered the composition of nails by affecting protein turnover but not gene expression in the nail matrix. 


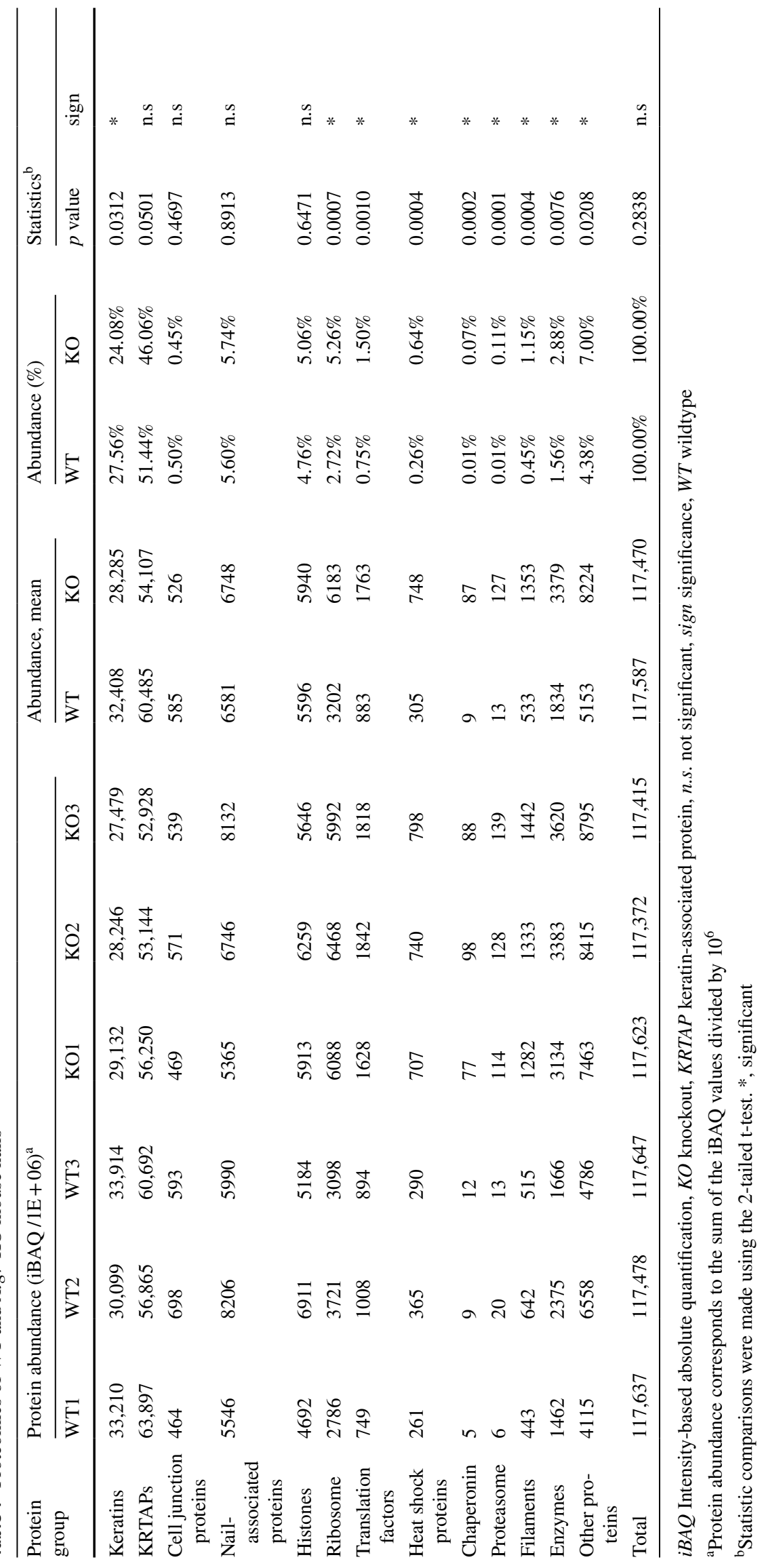


a

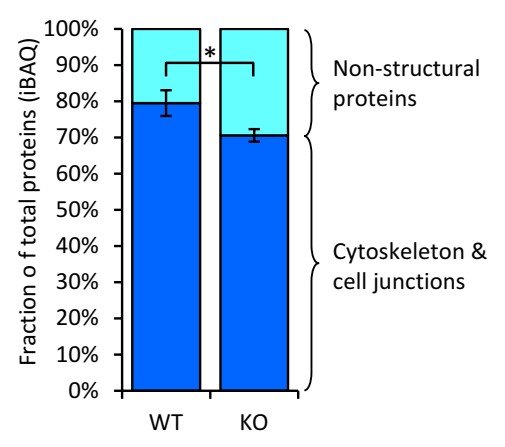

C

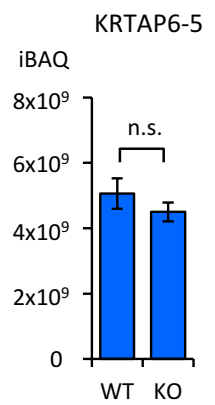

f

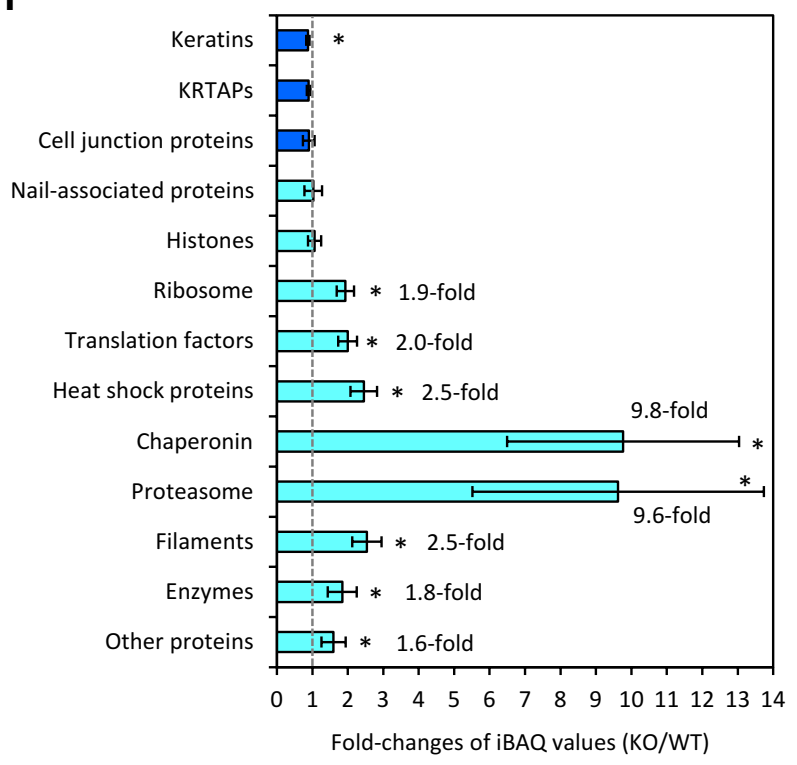

Fig. 3 Knockout of Atg7 in keratinocytes leads to significantly increased abundance of non-structural proteins in cornified nails. a iBAQ values of structural proteins (keratins, KRTAPs, cell junctions) (dark blue) and other proteins (light blue) in WT and KO nails. b-d iBAQ values of MAP1LC3A, KRTAP6-5, and DNase1L2 in WT and KO nails. e Real-time PCR quantification of DNase1L2 mRNAs in the nail matrix of WT and KO mice (a.u., arbitrary units). The graphs a-e show the means ( $n=3$ per genotype) and the error bars indicate standard deviations. *, $p<0.05$ (t-test); n.s., not significant. f Foldchanges of BBAQ values corresponding to groups of proteins in $\mathrm{KO}$ versus WT nails. The vertical grey dotted line marks a fold-change of 1 (equal abundance in WT and KO nails). The graph shows values calculated from means and the error bars indicate standard deviations. (Color figure online)

\section{Subunits of the proteasome and chaperonin are most strongly elevated upon genetic suppression of epithelial autophagy}

We further classified proteins according to their roles in nail matrix cells (e.g., ribosomal proteins, translation factors [translation initiation and elongation proteins], proteasomal proteins, and enzymes) and compared the abundance (iBAQ values) of these protein groups in $\mathrm{WT}$ and $\mathrm{KO}$ nails. Keratins and KRTAP were the predominant proteins in both WT and KO nails but their share of total peptides detected (iBAQ) was reduced by $3.5 \%$ and $5.4 \%$, respectively, in KO nails (Table 1) (Suppl. Fig. S5). This corresponded to fold-changes of 0.87 and 0.90 , respectively (Fig. 3f). Histones and "nail-associated proteins" LRRC15 and LYG1, expressed in the nail matrix but not in the epidermis of the sole (Suppl. Fig. S4), were the next most abundant protein groups. The iBAQ values of these proteins were not significantly different in WT and KO nail samples (Table 1; Fig. 3f).

The decrease in the abundance of keratins and KRTAPs was balanced by increases in the abundance of 8 groups of non-structural proteins: Ribosomal proteins, translation factors, chaperones, chaperonin, proteasomal proteins, filament proteins (microfilaments, microtubules, and associated proteins), enzymes, and the remaining other proteins increased by at least $50 \%$ of their respective iBAQ value when autophagy was suppressed, and the increases were significant $(p<0.05)$ for all these protein groups, hereafter referred to as putative autophagy substrates.

Ribosomal proteins were present at higher abundance than any other type of putative autophagy substrates in WT nails, and at even at higher abundance in KO nails (Table 1). The genetic suppression of autophagy increased their share (based on iBAQ) of the total proteins from 2.7 to $5.3 \%$, representing a 1.9-fold elevation (Fig. 3f). The finding that 73 of 76 detectable ribosomal proteins yielded higher iBAQ values in KO than WT nails confirmed the significance of this change. The largest group of putative autophagy substrates, according to our classification, were the enzymes. Among 177 enzymes detected in at least one of the nail samples, 155 (83\% of all enzymes) were elevated in the absence of keratinocyte autophagy (Fig. 4).

Among the proteins accumulating in nails of $\mathrm{KO}$ mice, the subunits of two large protein complexes stood out by particularly strong increases: the subunit proteins of CCT/TRiC chaperonin, which increased 9.8-fold and the protein components of proteasomes, which increased 9.6-fold (Fig. 3f). 8 of 8 chaperonin components and 28 of 28 detectable proteasome components were present at increased levels when autophagy was suppressed (Fig. 5a, b). The proteasome and the CCT/TRiC chaperonin were previously reported as preferential substrates of basal autophagy in cultured cells [36]. 
Fig. 4 Cornified nails of Atg7 KO mice contain aberrantly high amounts of functionally diverse enzymes. The graph shows the abundance of enzymes that have a mean iBAQ value of at least $10^{7}$ in either WT (black bars) or KO (red bars) nails ( $\mathrm{n}=3$ per genotype). Error bars indicate standard deviations. ${ }^{*}, p<0.05$; $* *, p<0.01$ (two-tailed t-test).

(Color figure online)

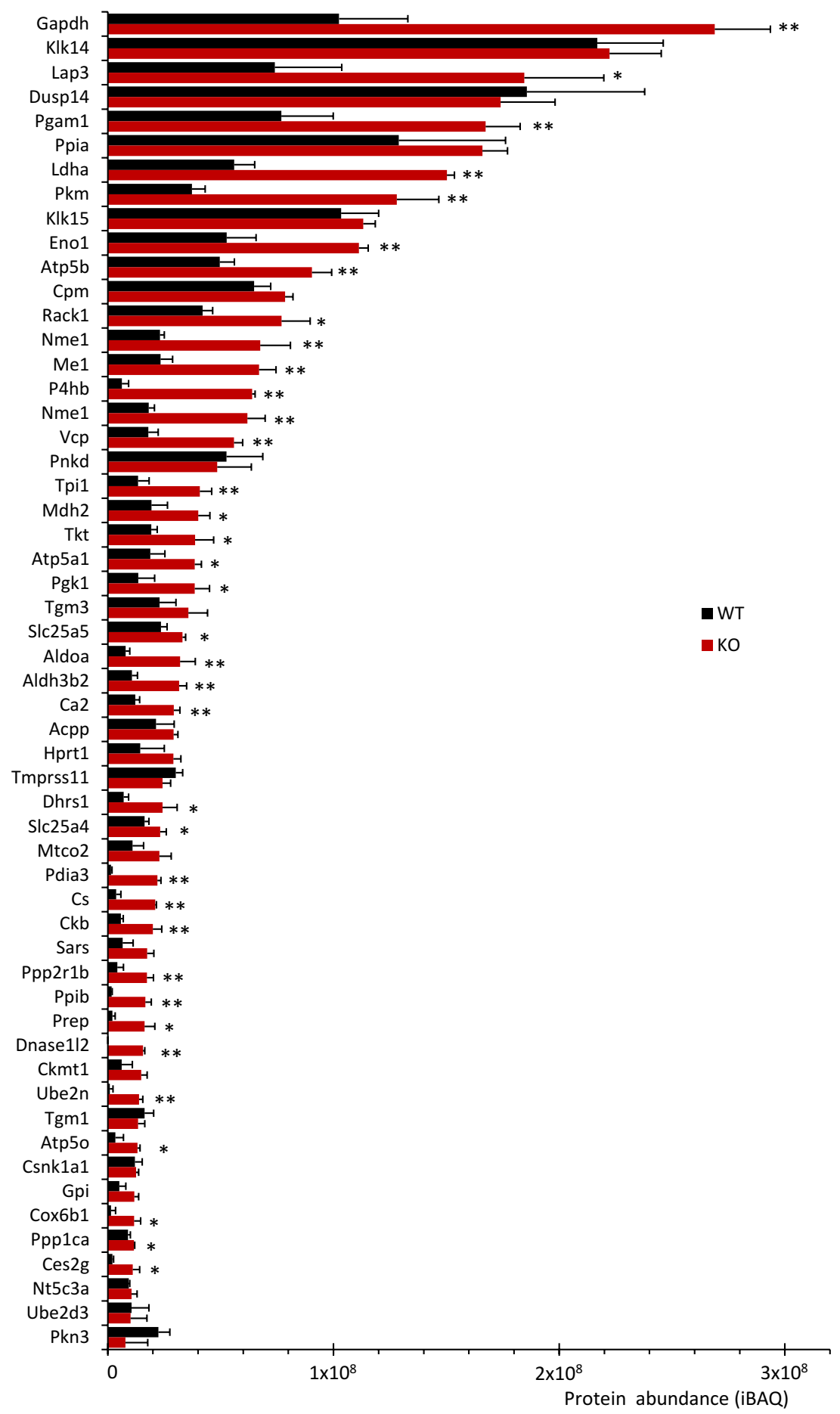

Thus, our results are compatible with the hypothesis that, in normal nail keratinocytes, a wide variety of nonstructural proteins is degraded by autophagy in a manner that is primarily determined by the accessibility of substrates, a process known as bulk autophagy (Fig. 5c), whereas deletion of $\operatorname{Atg} 7$ suppresses this process and leads to aberrant retention of non-structural proteins in nails of KO mice.

\section{Discussion}

The results of this study suggest that autophagy contributes to the intracellular remodeling during cornification of nail keratinocytes and provide new insights into the substrate specificity of autophagy in vivo. We utilized nail cornification as a model system because the products of this type of cornification, i.e. nails, can be prepared in pure form by 
Fig. 5 Proteasomal proteins and all subunits of the CCT chaperonin accumulate in cornified nails of $\operatorname{Atg} 7 \mathrm{KO}$ mice. a iBAQ values of proteasome subunits in WT (black bars) and $\mathrm{KO}$ (red bars) nails. b iBAQ values of chaperonin subunits in WT (black bars) and KO (red bars) nails. Error bars in a and $\mathbf{b}$ indicate standard deviations. *, $p<0.05 ; * *, p<0.01$ (two-tailed t test). c Schematic model of autophagic degradation of non-structural proteins in cornifying keratinocytes. (Color figure online) a

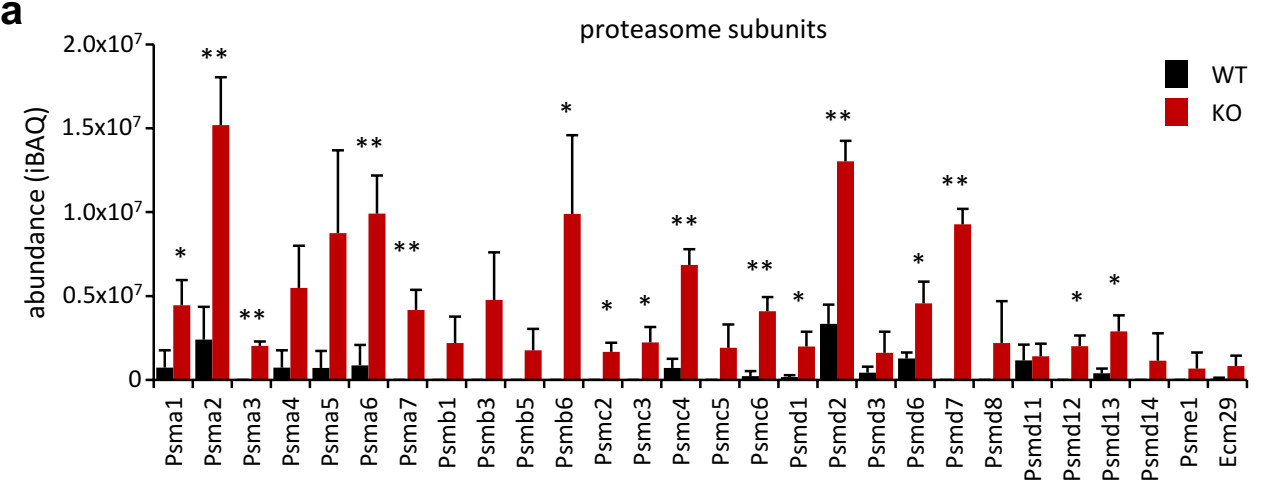

b

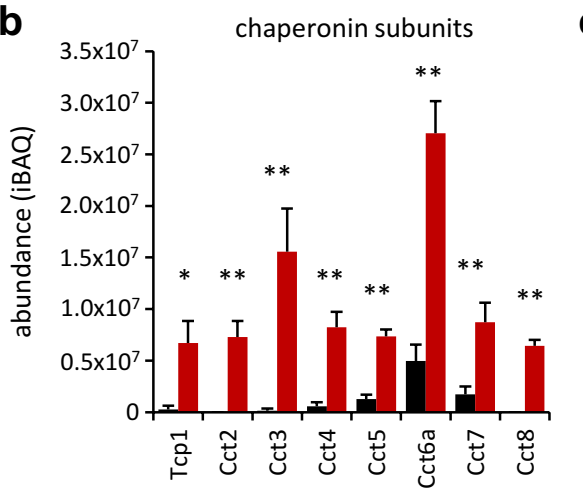

C

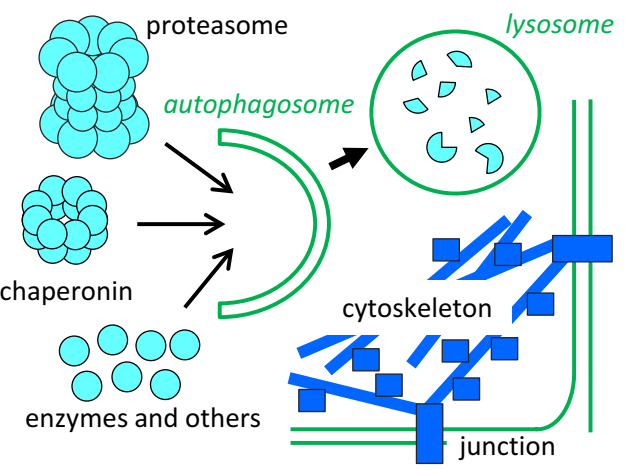

removing non-cornified material, and remain stable during storage prior to analysis. Accordingly, the variation between mass spectrometry results of nail samples from mice of the same genotype was low and the identification of autophagydependent changes in the abundance of numerous proteins was possible.

The deletion of the essential autophagy gene $\operatorname{Atg} 7$ in epithelial cells of $\mathrm{Atg} 7^{f l f} \mathrm{~K} 14-\mathrm{Cre}$ (KO) mice was demonstrated in previous studies $[14,17]$. Here, we confirmed the expectation that expression of $\mathrm{Atg} 7$ is also abrogated in the nail matrix (Fig. 2a), where keratinocytes are converted by cornification into building blocks of the nail. It is likely that autophagy is normally active in more than one or even in all differentiation stages (from the K14-positive stem cell stage to cornification) and, consequently, disturbances in protein turnover accumulate and lead to an altered nail proteome in KO mice. In agreement with the concept of an autophagydependent proteome phenotype, changes in the abundance of several nail proteins were not caused by changes in the levels of the corresponding mRNAs in the nail matrix (Suppl. Fig. S4).

Non-structural proteins of many functions from the synthesis of proteins to catalysis of metabolic reactions were elevated in nails of $\mathrm{KO}$ mice, arguing against a receptordependent specificity of autophagy during nail keratinocyte differentiation. Previous proteomics-based screening for autophagy substrates have provided evidence both for bulk degradation of cytoplasmic proteins (bulk autophagy), similar to the broad spectrum of functionally unrelated enzymes in our study (Fig. 4), and for substrate-specific receptormediated autophagy (selective autophagy) [37-42]. In nail keratinocytes, some proteins were more strongly affected by autophagy (or its absence) than others. The multiprotein complexes of the CCT chaperonin and the proteasome displayed the highest relative accumulation in the absence of autophagy. These results are in agreement with findings of a protein flux study [36] in which the proteasome and the CCT/TCP-1/TRiC chaperonin were demonstrated to be substrates of basal autophagy in cultured cells. Thus our study provides in vivo support for a hypothesis raised by in vitro data. Interestingly, autophagic degradation of proteasomes was recently shown to be an evolutionarily conserved process [43].

The turnover of large protein complexes such as chaperonin and the proteasome depends perhaps entirely on autophagy whereas many isolated proteins are degraded by both Atg7-dependent autophagy and proteasomes. Therefore, suppression of autophagy leads to particularly strong accumulations of large protein complexes in Atg7 KO nails and variable accumulations of other proteins depending on the accessibilities to autophagosomes and on the existence of alternative modes of breakdown for particular proteins. We propose that nail keratinocyte cornification employs bulk autophagy without signs of substrate-specific 
receptor-mediated degradation mechanisms. However, the precise degradation pathways of individual keratinocyte proteins remain to be dissected.

The elevations of proteasomes, chaperonin, and ribosomes in the absence of autophagy do not necessarily indicate increased activities of these molecular machines in differentiating keratinocytes and certainly not in corneocytes. However, it is at least conceivable that increased proteasomal degradation of specific proteins contributes to the proteome imbalance observed in Atg7 KO nails. In this regard it is interesting that keratins and KRTAPs are substrates of proteasomes $[44,45]$ and, accordingly, elevation of proteasomal activity may decrease the amounts of keratins in $\mathrm{KO}$ keratinocytes and nails. The increased concentration of large protein complexes, in particular ribosomes, may also influence the intracellular milieu independently of their activity. A twofold difference in the concentration of ribosomes, corresponding to the increase of ribosomal protein abundance in Atg7 KO corneocytes (Fig. 3f), was shown to enhance protein phase separation in the cytoplasm [46], a physical process likely relevant for cornification [47].

The decrease in the keratin and KRTAP fraction is perhaps the physiologically most important phenotype of the cornified nails in Atg7 KO mice. Keratins must be spared from autophagic degradation processes in WT nail keratinocytes to facilitate their function as cytoskeletal proteins while other proteins become dispensable, if not obstructive, in corneocytes. The activation of autophagy prior to death of cultured cells was previously shown to be compatible with the maintenance of the cytoskeleton whereas apoptosis led to the breakdown of cytoskeletal proteins [48]. Similarly, our results show that cytoskeletal proteins are maintained during autophagic protein turnover in cornifying keratinocytes in vivo and, in line with other articles $[2,49,50]$, refute the hypothesis that cornification of keratinocytes is related to apoptosis. In Atg7 KO nails, the cytoskeletal protein content is reduced due to the incomplete degradation of nonstructural proteins and possibly due to enhanced breakdown (via proteasomes) of keratins and KRTAPs. Keratins and associated proteins are the predominant material component of nails and the partial replacement of these proteins by non-structural proteins may cause defects in hardness of nails. However, under normal housing conditions there was no overt change in the shape of nails that would indicate increased abrasion or mechanically induced deformation in Atg7 KO mice (Suppl. Fig. S1). As mouse nails are too small and irregular in shape for standard assays of material hardness, sophisticated in vitro measurements or in vivo stress tests of nails remain to be applied to test whether autophagy contributes substantially to the hardening of the nail during cornification. It will also be interesting to subject the stratum corneum of WT and KO mice to comprehensive comparative proteomics and to perform stress tests that go beyond the basic characterization performed previously [14].

This study utilized nails primarily as a model system of cornification with many features advantageous for proteomics. Besides providing new insights into the mechanisms of intracellular remodeling during keratinocyte cornification, the results reported here may also have implications on therapies and diagnosis of skin diseases. Drugs that suppress autophagy, such as hydroxychloroquine, and drugs that increase autophagic activity, such as mTOR inhibitors, are currently being used or tested for the treatment of a variety of skin diseases including some with cornification abnormalities [51, 52]. Inhibitors of mTOR, which affect autophagy and other processes, are associated with nail dystrophy [53], pointing to dysregulation of cornificationassociated autophagy as a potentially relevant side-effect of these drugs. In patients with psoriasis, keratinocytes forming the epidermal stratum corneum are affected by deregulation of autophagy $[13,54]$, suggesting that modulation of autophagy might be used in therapies of skin inflammation or cornification defects. Finally, our demonstration that suppression of autophagy correlates with changes in nail protein composition indicates that proteomic analysis of nails, and perhaps other cornified epidermal structures, may be developed further to uncover disease-associated aberrations in protein turnover for diagnostic applications.

In conclusion, this study suggests that autophagy is active during normal nail formation and that suppression of autophagy alters the proteome of cornified nails with differential effects on cytoskeletal and non-cytoskeletal proteins.

Acknowledgements Open access funding provided by Medical University of Vienna. The authors thank Harald Höger, Core Unit of Biomedical Research, Medical University of Vienna for the maintenance of the mice and the Core Facility Imaging, Medical University Vienna for excellent support. We also thank Heidemarie Rossiter for helpful comments and Richard Eigenheer for expert mass spectrometric assistance. Atg7-floxed mice were kindly provided by Prof. Masaaki Komatsu, Tokyo Metropolitan Institute of Medical Science, Tokyo, Japan.

\section{Compliance with ethical standards}

Conflict of interest The authors declare that they have no conflict of interest.

Ethical approval All applicable international, national, and/or institutional guidelines for the care and use of animals were followed.

Open Access This article is distributed under the terms of the Creative Commons Attribution 4.0 International License (http://creativeco mmons.org/licenses/by/4.0/), which permits unrestricted use, distribution, and reproduction in any medium, provided you give appropriate credit to the original author(s) and the source, provide a link to the Creative Commons license, and indicate if changes were made. 


\section{References}

1. Candi E, Schmidt R, Melino G (2005) The cornified envelope: a model of cell death in the skin. Nat Rev Mol Cell Biol 6:328-340

2. Eckhart L, Lippens S, Tschachler E, Declercq W (2013) Cell death by cornification. Biochim Biophys Acta 1833:3471-3480

3. Fleckman P, Jaeger K, Silva KA, Sundberg JP (2013) Comparative anatomy of mouse and human nail units. Anat Rec (Hoboken) 296:521-532

4. Jones LA, Harland DP, Jarrold BB, Connolly JE, Davis MG (2018) The walking dead: sequential nuclear and organelle destruction during hair development. Br J Dermatol 178:1341-1352

5. Mariño G, López-Otín C (2004) Autophagy: molecular mechanisms, physiological functions and relevance in human pathology. Cell Mol Life Sci 61:1439-1454

6. Mizushima N, Levine B (2010) Autophagy in mammalian development and differentiation. Nat Cell Biol 12:823-830

7. Mizushima N, Komatsu M (2011) Autophagy: renovation of cells and tissues. Cell 47:728-741

8. Choi AM, Ryter SW, Levine B (2013) Autophagy in human health and disease. N Engl J Med 368:651-662

9. Haruna K, Suga Y, Muramatsu S et al (2008) Differentiationspecific expression and localization of an autophagosomal marker protein (LC3) in human epidermal keratinocytes. J Dermatol Sci $52: 213-215$

10. Sukseree S, Eckhart L, Tschachler E, Watanapokasin R (2013) Autophagy in epithelial homeostasis and defense. Front Biosci E5:1000-1010

11. Moriyama M, Moriyama H, Uda J, Matsuyama A, Osawa M, Hayakawa T (2014) BNIP3 plays crucial roles in the differentiation and maintenance of epidermal keratinocytes. J Investig Dermatol 134:1627-1635

12. Yoshihara N, Ueno T, Takagi A et al (2015) The significant role of autophagy in the granular layer in normal skin differentiation and hair growth. Arch Dermatol Res 307:159-169

13. Akinduro O, Sully K, Patel A et al (2016) Constitutive autophagy and nucleophagy during epidermal differentiation. J Investig Dermatol 136:1460-1470

14. Rossiter H, König U, Barresi C et al (2013) Epidermal keratinocytes form a functional skin barrier in the absence of Atg7 dependent autophagy. J Dermatol Sci 71:67-75

15. Sukseree S, Rossiter H, Mildner M et al (2013) Targeted deletion of Atg5 reveals differential roles of autophagy in keratin K5-expressing epithelia. Biochem Biophys Res Commun 430:689-694

16. Fischer H, Fumicz J, Rossiter $\mathrm{H}$ et al (2017) Holocrine secretion of sebum is a unique DNase2-dependent mode of programmed cell death. J Investig Dermatol 137:587-594

17. Sukseree S, Mildner M, Rossiter H et al (2012) Autophagy in the thymic epithelium is dispensable for the development of selftolerance in a novel mouse model. PLoS ONE 7:e38933

18. Sukseree S, Bergmann S, Pajdzik K et al (2018) Suppression of epithelial autophagy compromises the homeostasis of sweat glands during aging. J Investig Dermatol 138:2061-2063

19. Sukseree S, Bergmann S, Pajdzik K, Tschachler E, Eckhart L (2018) Suppression of autophagy perturbs turnover of sequestosome-1/p62 in Merkel cells but not in keratinocytes. J Dermatol Sci 90:209-211

20. Rossiter H, Stübiger G, Gröger M et al (2018) Inactivation of autophagy leads to changes in sebaceous gland morphology and function. Exp Dermatol 27:1142-1151

21. Zhao Y, Zhang CF, Rossiter H et al (2013) Autophagy is induced by UVA and promotes removal of oxidized phospholipids and protein aggregates in epidermal keratinocytes. J Investig Dermatol 133:1629-1637
22. Komatsu M, Waguri S, Ueno T et al (2005) Impairment of starvation-induced and constitutive autophagy in Atg7-deficient mice. J Cell Biol 169:425-434

23. Mizushima N, Yamamoto A, Matsui M, Yoshimori T, Ohsumi Y (2004) In vivo analysis of autophagy in response to nutrient starvation using transgenic mice expressing a fluorescent autophagosome marker. Mol Biol Cell 15:1101-1111

24. Manils J, Fischer H, Climent J et al (2017) Double deficiency of Trex2 and Dnase1L2 nucleases leads to accumulation of DNA in lingual cornifying keratinocytes without activating inflammatory responses. Sci Rep 7:11902

25. Fischer H, Rossiter H, Ghannadan M et al (2005) Caspase-14 but not caspase-3 is processed during the development of fetal mouse epidermis. Differentiation 73:406-413

26. Rice RH, Means GE, Brown WD (1977) Stabilization of bovine trypsin by reductive methylation. Biochim Biophys Acta 492:316-321

27. Nesvizhskii AI, Keller A, Kolker E, Aebersold RA (2003) A statistical model for identifying proteins by tandem mass spectrometry. Anal Chem 75:4646-4658

28. Schwanhäusser B, Busse D, Li N et al (2011) Global quantification of mammalian gene expression control. Nature 473:337-342

29. Song X, Narzt MS, Nagelreiter IM et al (2017) Autophagy deficient keratinocytes display increased DNA damage, senescence and aberrant lipid composition after oxidative stress in vitro and in vivo. Redox Biol 11:219-230

30. Zhang CF, Gruber F, Ni C et al (2015) Suppression of autophagy dysregulates the antioxidant response and causes premature senescence of melanocytes. J Investig Dermatol 135:1348-1357

31. Pfaffl MW (2001) A new mathematical model for relative quantification in real-time RT-PCR. Nucleic Acids Res 29:e45

32. Jäger K, Fischer H, Tschachler E, Eckhart L (2007) Terminal differentiation of nail matrix keratinocytes involves up-regulation of DNase1L2 but is independent of caspase-14 expression. Differentiation 75:939-946

33. Fischer H, Szabo S, Scherz J et al (2011) Essential role of the keratinocyte-specific endonuclease DNase1L2 in the removal of nuclear DNA from hair and nails. J Investig Dermatol 131:1208-1215

34. Rice RH, Xia Y, Alvarado RJ, Phinney BS (2010) Proteomic analysis of human nail plate. J Proteome Res 9:6752-6758

35. Rice RH, Bradshaw KM, Durbin-Johnson BP et al (2013) Distinguishing ichthyoses by protein profiling. PLoS ONE 8:e75355

36. Zhang T, Shen S, Qu J, Ghaemmaghami S (2016) Global analysis of cellular protein flux quantifies the selectivity of basal autophagy. Cell Rep 14:2426-2639

37. Cudjoe EK Jr, Saleh T, Hawkridge AM, Gewirtz DA (2017) Proteomics insights into autophagy. Proteomics 17:1700022

38. Wong YK, Zhang J, Hua ZC, Lin Q, Shen HM, Wang J (2017) Recent advances in quantitative and chemical proteomics for autophagy studies. Autophagy 13:1472-1486

39. Mancias JD, Wang X, Gygi SP, Harper JW, Kimmelman AC (2014) Quantitative proteomics identifies NCOA4 as the cargo receptor mediating ferritinophagy. Nature 509:105-109

40. Mathew R, Khor S, Hackett SR, Rabinowitz JD, Perlman DH, White E (2014) Functional role of autophagy-mediated proteome remodeling in cell survival signaling and innate immunity. Mol Cell 55:916-930

41. Matsumoto N, Ezaki J, Komatsu M et al (2008) Comprehensive proteomics analysis of autophagy-deficient mouse liver. Biochem Biophys Res Commun 368:643-649

42. Zhuo C, Ji Y, Chen Z et al (2013) Proteomics analysis of autophagy-deficient Atg7-/- MEFs reveals a close relationship between F-actin and autophagy. Biochem Biophys Res Commun 437:482-488 
43. Marshall RS, Vierstra RD (2018) To save or degrade: balancing proteasome homeostasis to maximize cell survival. Autophagy 14:2029-2031

44. Ku NO, Omary MB (2000) Keratins turn over by ubiquitination in a phosphorylation-modulated fashion. J Cell Biol 149:547-552

45. Fujimoto S, Takase T, Kadono N, Maekubo K, Hirai Y (2014) Krtap11-1, a hair keratin-associated protein, as a possible crucial element for the physical properties of hair shafts. J Dermatol Sci 74:39-47

46. Delarue M, Brittingham GP, Pfeffer S et al (2018) mTORC1 controls phase separation and the biophysical properties of the cytoplasm by tuning crowding. Cell 174:338-349

47. McKinnon AJ, Harland DP (2011) A concerted polymerizationmesophase separation model for formation of trichocyte intermediate filaments and macrofibril templates. 1: relating phase separation to structural development. J Struct Biol 173:229-240

48. Bursch W, Hochegger K, Torok L, Marian B, Ellinger A, Hermann RS (2000) Autophagic and apoptotic types of programmed cell death exhibit different fates of cytoskeletal filaments. J Cell Sci 113:1189-1198
49. Lippens S, Denecker G, Ovaere P, Vandenabeele P, Declercq W (2005) Death penalty for keratinocytes: apoptosis versus cornification. Cell Death Differ 12:1497-1508

50. Lippens S, Hoste E, Vandenabeele P, Agostinis P, Declercq W (2009) Cell death in the skin. Apoptosis 14:549-569

51. Tschachler E, Eckhart L (2017) Autophagy—how to control your intracellular diet. Br J Dermatol 176:1417-1419

52. Manousaridis I, Manousaridis K, Peitsch WK, Schneider SW (2013) Individualizing treatment and choice of medication in lichen planus: a step by step approach. J Dtsch Dermatol Ges 11:981-991

53. Peuvrel L, Quéreux G, Brocard A, Saint-Jean M, Dréno B (2012) Onychopathy induced by temsirolimus, a mammalian target of rapamycin inhibitor. Dermatology 224:204-208

54. Mahil SK, Twelves S, Farkas K et al (2016) AP1S3 mutations cause skin autoinflammation by disrupting keratinocyte autophagy and up-regulating IL-36 production. J Investig Dermatol 136:2251-2259 